\title{
Flight Control Role in RLV Configuration Development ${ }^{\dagger}$
}

\author{
Dr. Hussein Youssef ${ }^{\S}$ \\ Howard Lee ${ }^{\ddagger}$ \\ Dr. Rajiv Chowdhry $\#$ \\ Chris Cotting \\ Lockheed Martin Aeronautics Company-Palmdale \\ Palmdale, CA 93599
}

\begin{abstract}
A new optimization technique was used to aide in the selection of aerodynamic surfaces and Thrust Vectoring Control (TVC) based on maximizing control margin during ascent and entry portions of the trajectory. In order to meet the mass fraction requirement of RLV, every substructure needed to be carefully designed to minimize the dry mass. The trajectory was designed to deliver the payload required for each mission subject to thermal and structural constraints. At each point on the trajectory the angle of attack and Mach number pair was used to calculate the control power required to trim and to stabilize the vehicle. The new optimization technique was based on finding the configuration that minimizes the control power peaks along the entire trajectory.
\end{abstract}

\section{BACKGROUND}

The key enabling technology in RLV program is to achieve mass fraction that is commercially viable. The minimum weight design has been researched heavily as a multidisciplinary optimization problem in the government research facilities and industry. What is more challenging in the RLV, the fixed geometry design, the variation of stability along the different Mach regimes, and the nonlinearity of the aerodynamics and control functions. A relaxed static stability design is the main ingredient in achieving minimum weight design. However, there is always a limit in how far the relaxed stability could be tolerated which is governed by how fast the actuation and how complex the control architecture would be.
Traditionally, a full multidisciplinary optimization would require a computational fluid dynamic (CFD) and finite element model tools which take so long to implement and to converge. Our philosophy is centered around two-tier approach. The first tier optimization is based on the coupling between performance and structure sizing. The second tier optimization is based on the coupling between control and external configuration which is the subject of this paper.

\section{INTRODUCTION}

One important factor in achieving our goal is the availability of wind tunnel data across all Mach regimes for different configurations. Although the wind tunnel data was not heavily populated, it was good enough to construct the partial derivatives of aerodynamic forces and moments with respect to geometrical changes. The variability of the geometry was accomplished through scaling of areas and the two angles of canted fin incidence and dihedral. The validity of simulation data in the neighborhood of actual wind tunnel data was considered adequate for gross aerodynamic effect since the interest was in the minimum control sizing and not aerodynamic shaping optimization. More details regarding optimization databases are described in section 3 .

The control function used all the effectors available in different phases of the mission. During ascent, the thrust vectoring control (TVC) of the engine and the control surfaces were used. During entry, the reaction control system (RCS) and the control surfaces were used. Along selected points on the trajectory, Mach and angle of attack (AOA) were identified in order to establish the basic untrimmed condition and the open

\footnotetext{
'This paper acknowledges NASA's support under Cooperative Agreement NCC8-115.

Technical Fellow, Sr. Member ALAt.

Sr. Research and Development Engineer.

"Research and Development Engineer, Member AIAA.

- Servo Analysis Engineer, Member AIAA
} 
loop stability. A trim requirement and closed loop stability target were established for both longitudinal and lateral axes. The control mixing scheme was chosen to reach saturation of all effectors at the same time. Control power requirements and mixing scheme are outlined in section 4 .

Different optimization algorithms were constructed based on different approaches of minimizing weight. The one that converges reasonably well was based on minimizing the maximum control deflections subject to constraining the size of aerodynamic surfaces. The optimization was carried out along the entire trajectory for different missions and abort scenario. The algorithms were implemented using Matlab constraint optimization toolbox. Section 5 describes the optimization formulation.

A converged optimization was achieved for several cases. All results obtained so far indicated a very promising achievement in minimum weight optimization. Specific results are shown in section 6 .

The next step of this effort is providing the following wind tunnel entry with configuration closer the optimized solution to confirm the validity the obtained results. Final conclusions are briefed in section 7 .

\section{OPTIMIZATION DATABASES}

Several databases were implemented to accomplish the optimization effort. They included aerodynamics, propulsion, RCS, and mass properties. The aerodynamics databases had a baseline configuration and several others that were perturbation to the baseline. Each element of the six forces and moments were tabulated as function of AOA $(\alpha)$, sideslip angle $(\beta)$, Mach (M), and control surface deflection ( $\delta$ ). For example, the pitching moment coefficient $\left(C_{m}\right)$ for the baseline was in constructed in the following form:

$$
C_{m_{\text {न }}}=f(\alpha, \beta, M, \delta)
$$

In addition, changes due to different configuration were generated using partial derivatives, e.g.

$$
C_{m}=C_{m m}+\frac{\partial C_{m}}{\partial A} \Delta A+\frac{\partial C_{m}}{\partial I} \Delta I+\ldots
$$

Where

$$
\begin{aligned}
& A=\text { Area of different canted fins } \\
& I=\text { Incidence angle of canted fin }
\end{aligned}
$$

In order for the algorithms to function properly, special effort were spent in smoothing out the data to remove sharp discontinuities. In addition, extrapolation capabilities were added to allow the optimizer to complete the search. However, the optimum solution was checked at the end to assure convergence without saturation for any effector. It was necessary to generate tables of six entries to take out any discontinuities, which caused the optimization to slow down. The six variables were Mach, $\alpha, \beta, A$, $I$, and body flap area. Control effectiveness was adjusted according to the variation of the surface area.

\section{CONTROL REQUIREMENTS AND MLXING}

The control power required to trim and stabilize the vehicle was derived for different effectors during each flight phase. The overall scheme of calculating the control power required is depicted in figure 1 . In the area where the open loop stability was below target, additional control was added to bring the closed loop stability to the acceptable level. The closed loop stability target was scheduled as function of Mach for both modes; the short period and the dutch roll. A deterministic wind disturbance was introduced to excite the unstable mode. Again the delta $\mathrm{AOA}$ and Beta disturbances were scheduled as function of Mach.

The calculation of control surfaces deflection to provide trim and stabilization were accomplished simultaneously using nonlinear equations solver. For example we started by closed loop stability of short period $\omega_{5 p}$. From that we can calculate $M_{\alpha}$ and hence q-dot required as a result of $\Delta \alpha$ perturbation. With proper control mixing, control effectors were calculated to satisfy the required torque. 


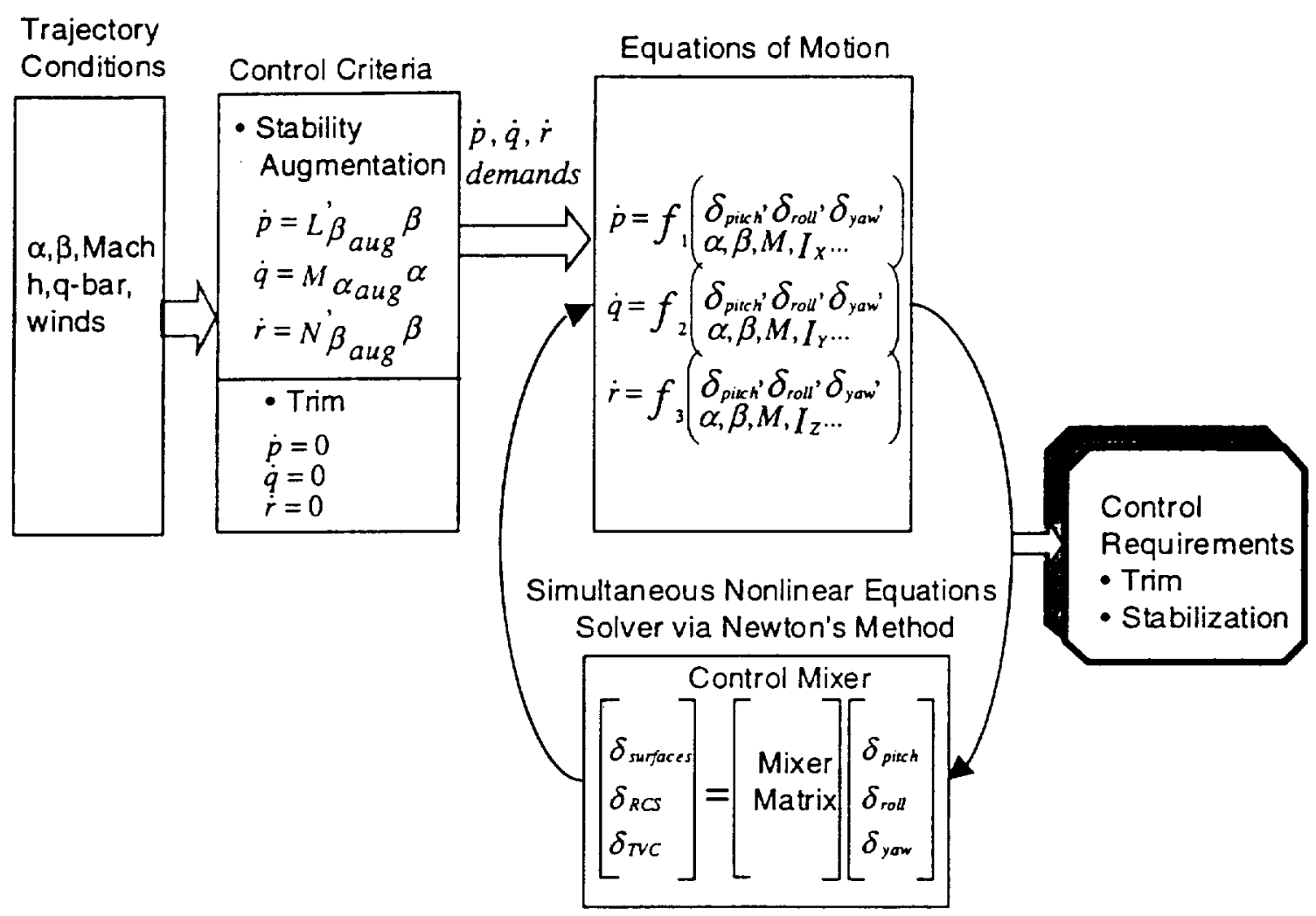

Figure 1 - Control Power Requirement Algorithm Overview

\section{OPTIMIZATION CRITERIA}

As a first step, the canted fin area and incidence angle was considered in the optimization. At selected points on the trajectory, three dimensional plots were generated to examine the percentage of effector usage as function of canted fin areas and incidence angle. Once it was determined that there was a range of canted fin area and incidence angle across the different Mach and AOA where there was enough control available to trim and stabilize the vehicle, the optimizer would be initiated from that feasible solution.

Different optimization strategies were implemented in an attempt to find a converged solution in a reasonable turn around time. The overall objective was to minimize the area subject to the constraint of not to exceed certain control deflections. However, it was easier to formulate the optimization the other way around:

$$
\min _{t}\left|\delta_{\max }\right| \text { subject } 10 A \leq a
$$

By parametrically varying the value of $a$, and solving the optimization problem, one could determine the area required to achieve acceptable level of control deflections to trim and stabilize the vehicle.

Later on where flap area was included in the optimization, the constraint was changed to total weight rather than area of each surface. The weight was calculated based on parametric unit weight for each surface: 


$$
W t=\sum W_{j} A_{j}
$$

So, the final optimization was formulated as follows:

$$
\min _{t}\left|\delta_{\max }\right| \quad \text { subject to } W t \leq w
$$

\section{RESULTS}

Considerable check cases were generated to validate the aerodynamic databases against the wind tunnel data at the break points. Open loop stability for longitudinal ( $\omega_{s p}$ short period) and lateral-directional ( $\omega_{d}$ dutch roll) axes were calculated and verified against static stability plots $\left(\mathrm{d} C_{m} / \mathrm{d} C_{N}\right.$.) A reference configuration, based on an initial canted fin size, incidence and body flap area, was used as a starting condition for the optimization. Figures 2 and 3 show open loop and closed loop stability targets for both axes during entry for the reference configuration. Figures 4 to 9 show control surface deflections used for trim and stabilization for this reference configuration along a typical reentry trajectory. Figures 10 to 13 show the feasibility plots at four different points on the entry trajectory where there were larger control activities. Using the reference configuration as a starting point for the optimizer, a minimum weight solution was obtained. Figures 14 to 19 show the final optimized configuration control surface deflections. The optimized configuration achieved reduction in total weight compared to the reference configuration while maintaining similar levels of control surface usage.

\section{CONCLUSIONS}

Successful optimization technique based on control deflection criteria was achieved to aid in the refinement of external configuration. The optimized configuration had the potential of $15 \%$ weight reduction compared to the reference configuration. Also, it provided striking balance between the relative sizes of the different surfaces. The real benefit was aiding in the selection of the component sizes for the next wind tunnel entry.

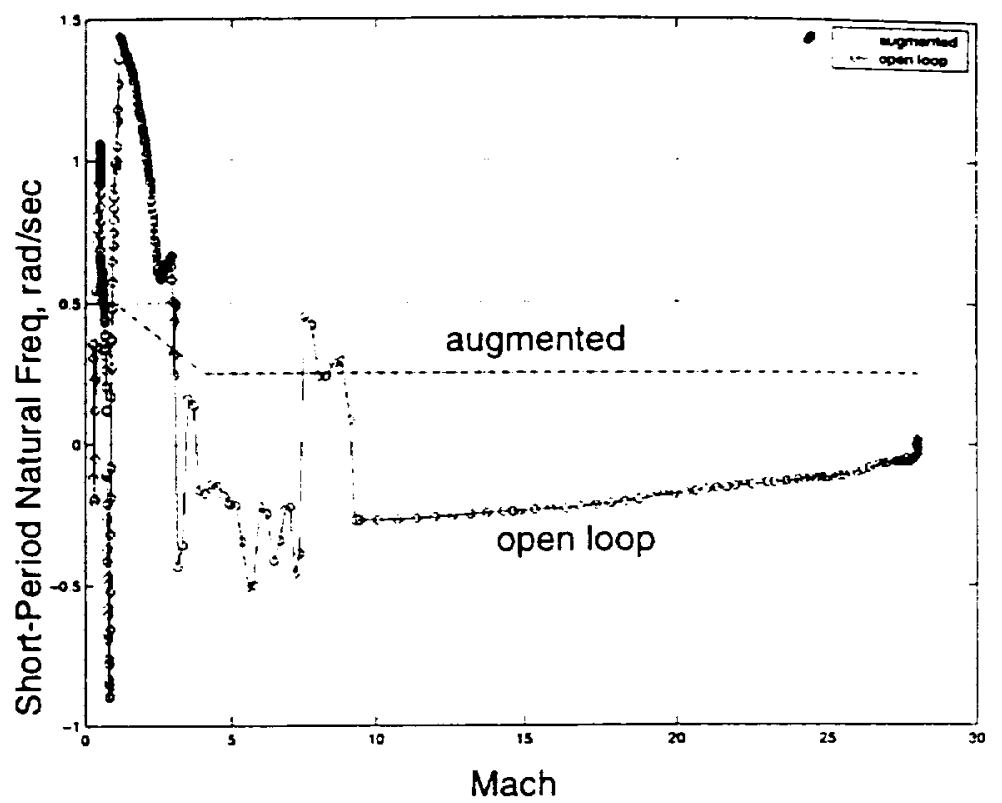

Figure 2- Open Loop Short-Period Characteristic of the Reference Configuration and Augmented Stability Target

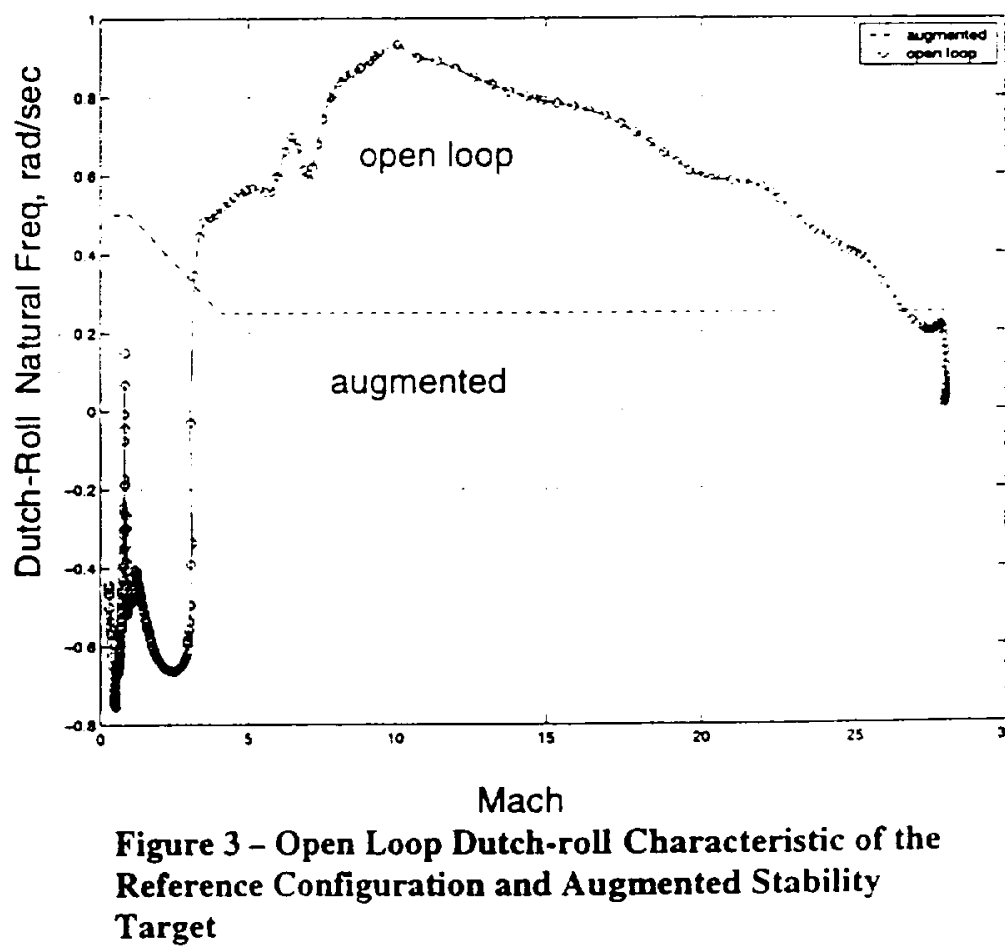




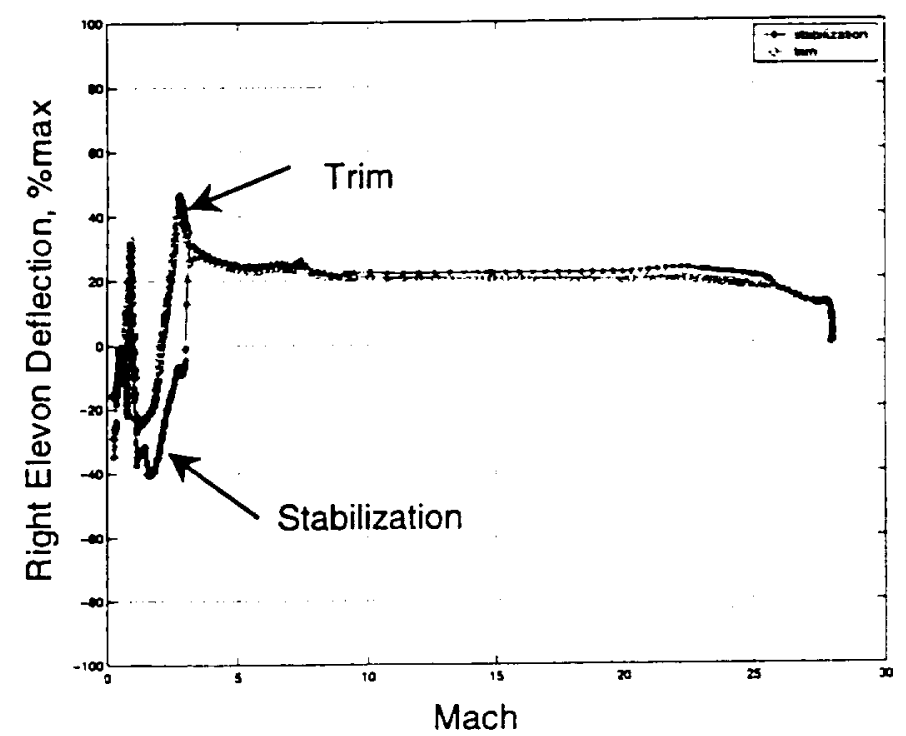

Figure 4 - Right Elevon Deflections Used for Trim and Stabilization for the Reference Configuration

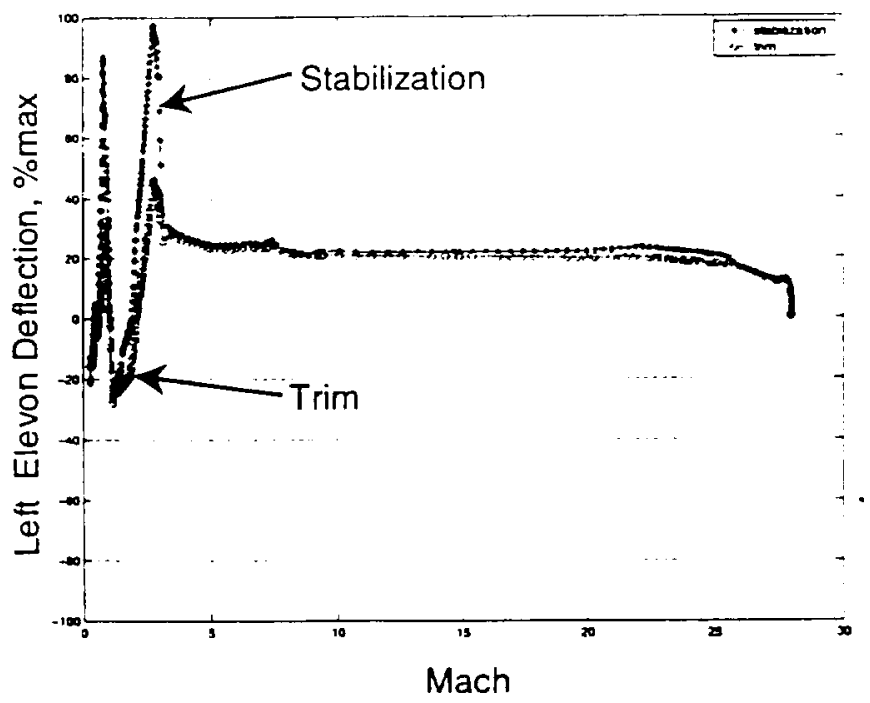

Figure 5 - Left Elevon Deflections Used for Trim and Stabilization for the Reference Configuration

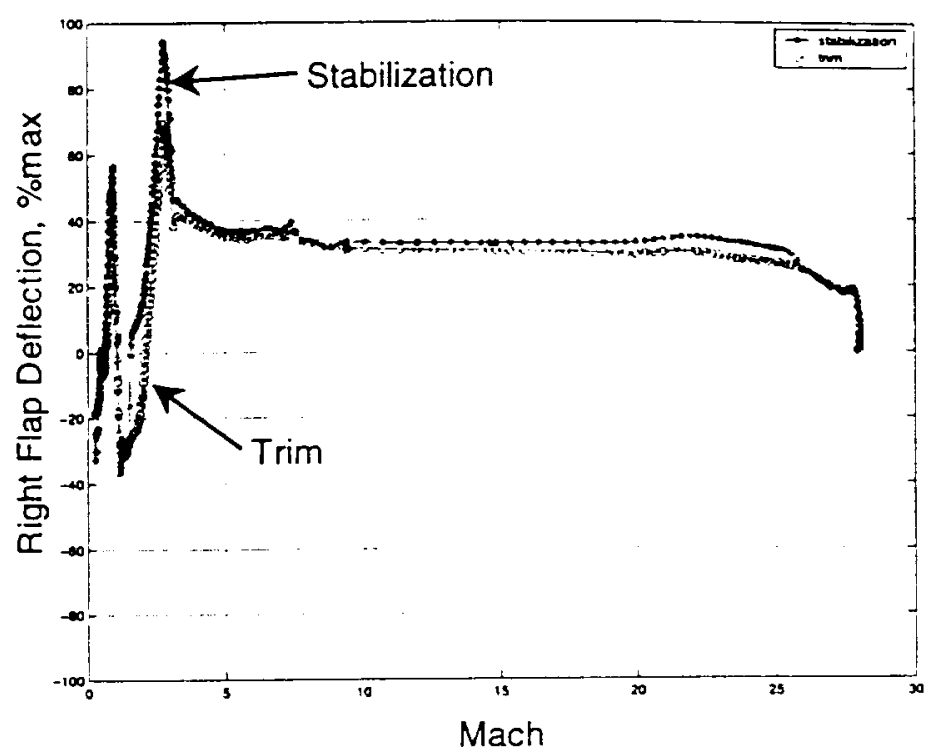

Figure 6 - Right Flap Deflections Used for Trim and Stabilization for the Reference Configuration

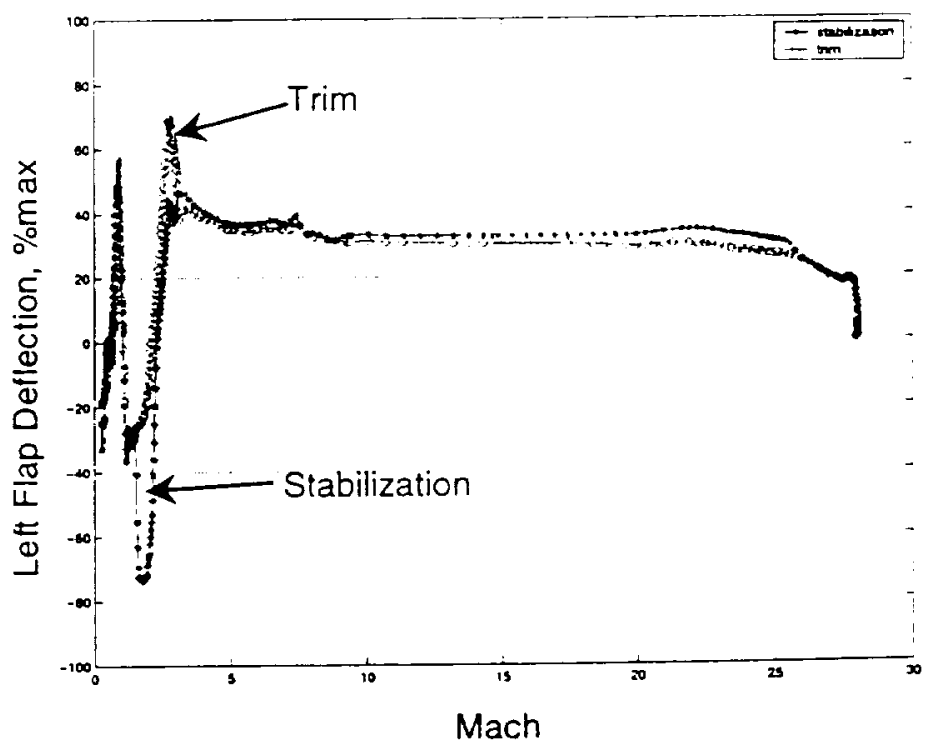

Figure 7 - Left Flap Deflections Used for Trim and Stabilization for the Reference Configuration 


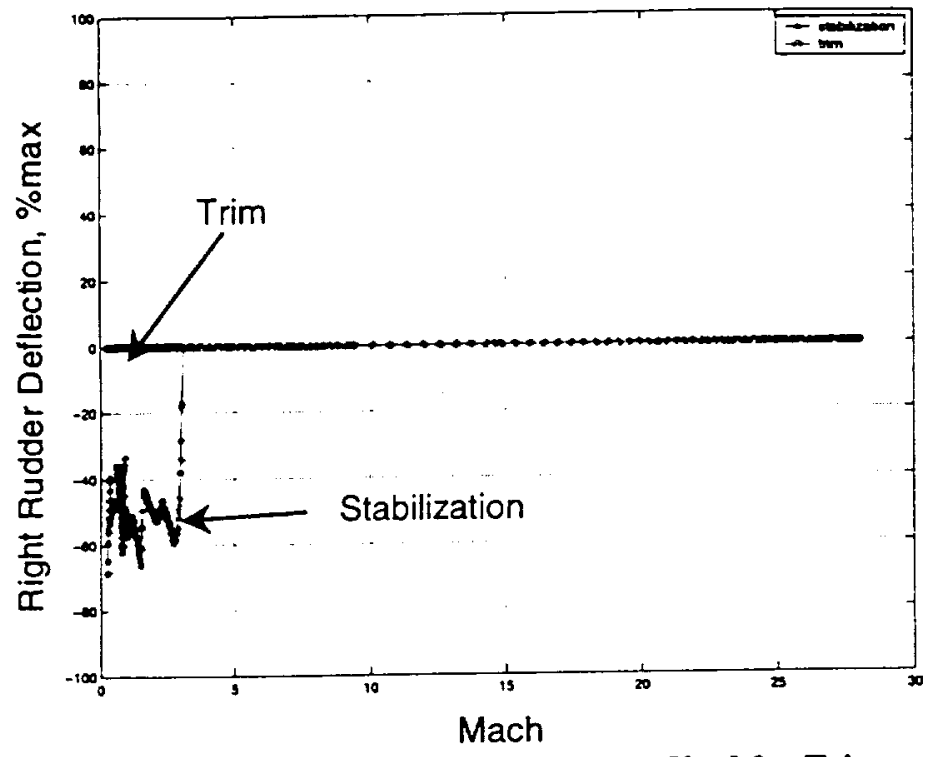

Figure 8 - Right Rudder Deflections Used for Trim and Stabilization for the Reference Configuration

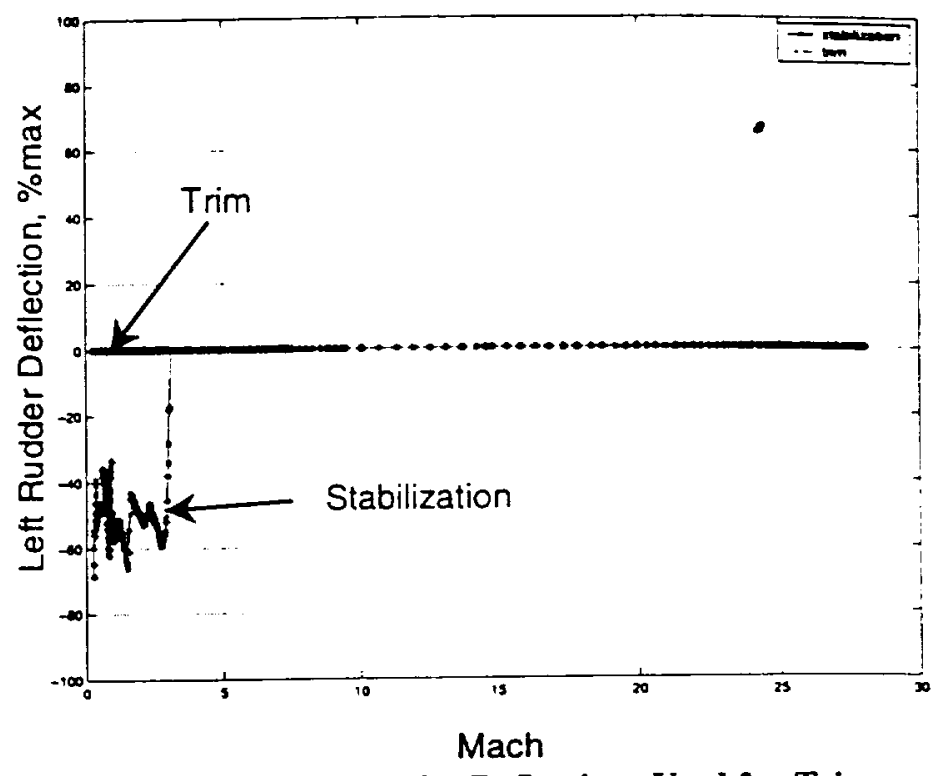

Figure 9 - Left Rudder Deflections Used for Trim and Stabilization for the Reference Configuration

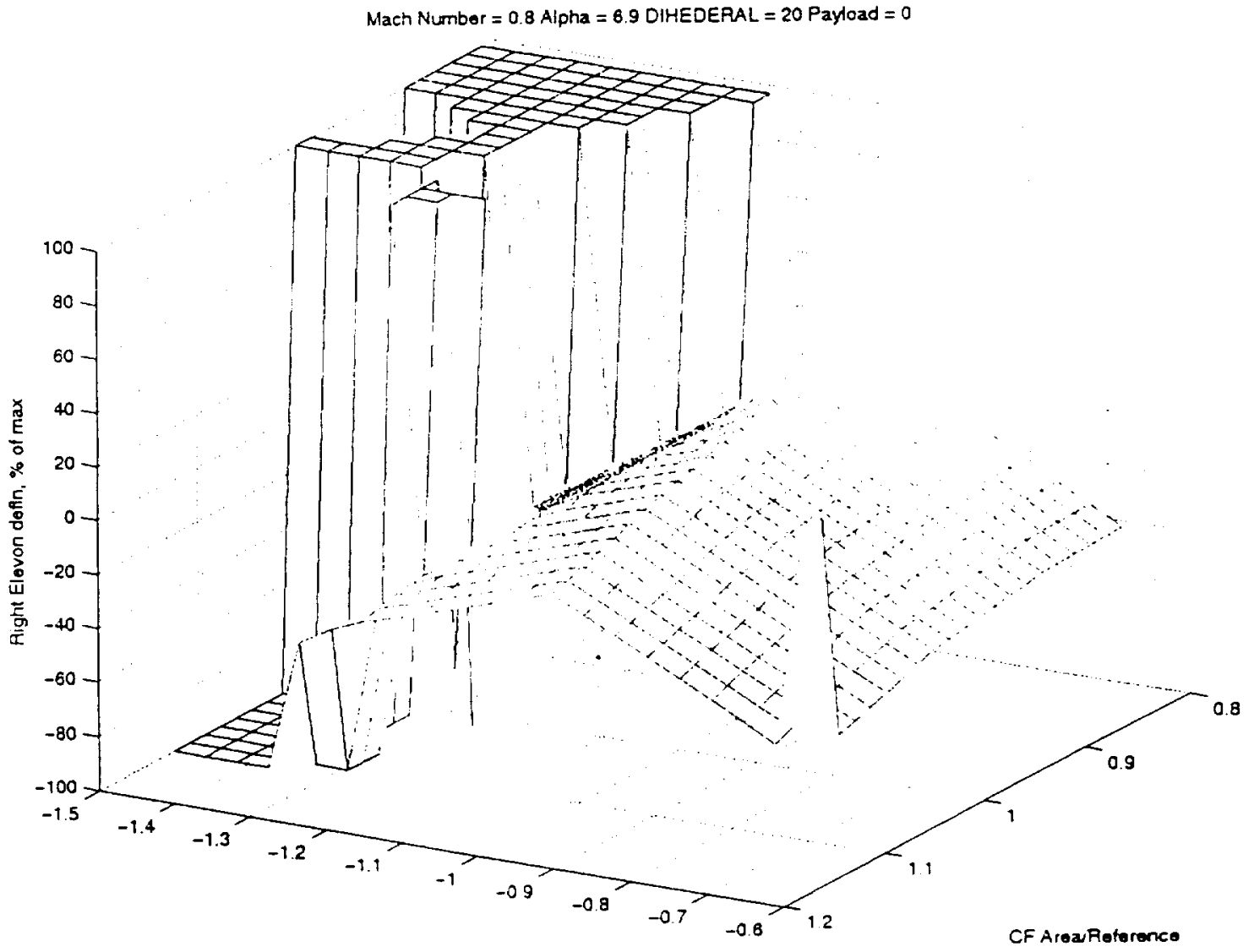

CF Incidences Referenco

Figure 10 - Right Elevon Deflections as Function of Canted Fin Area and Incidence at Mach $=0.8$ and Alpha $=6.9 \mathrm{deg}$ 


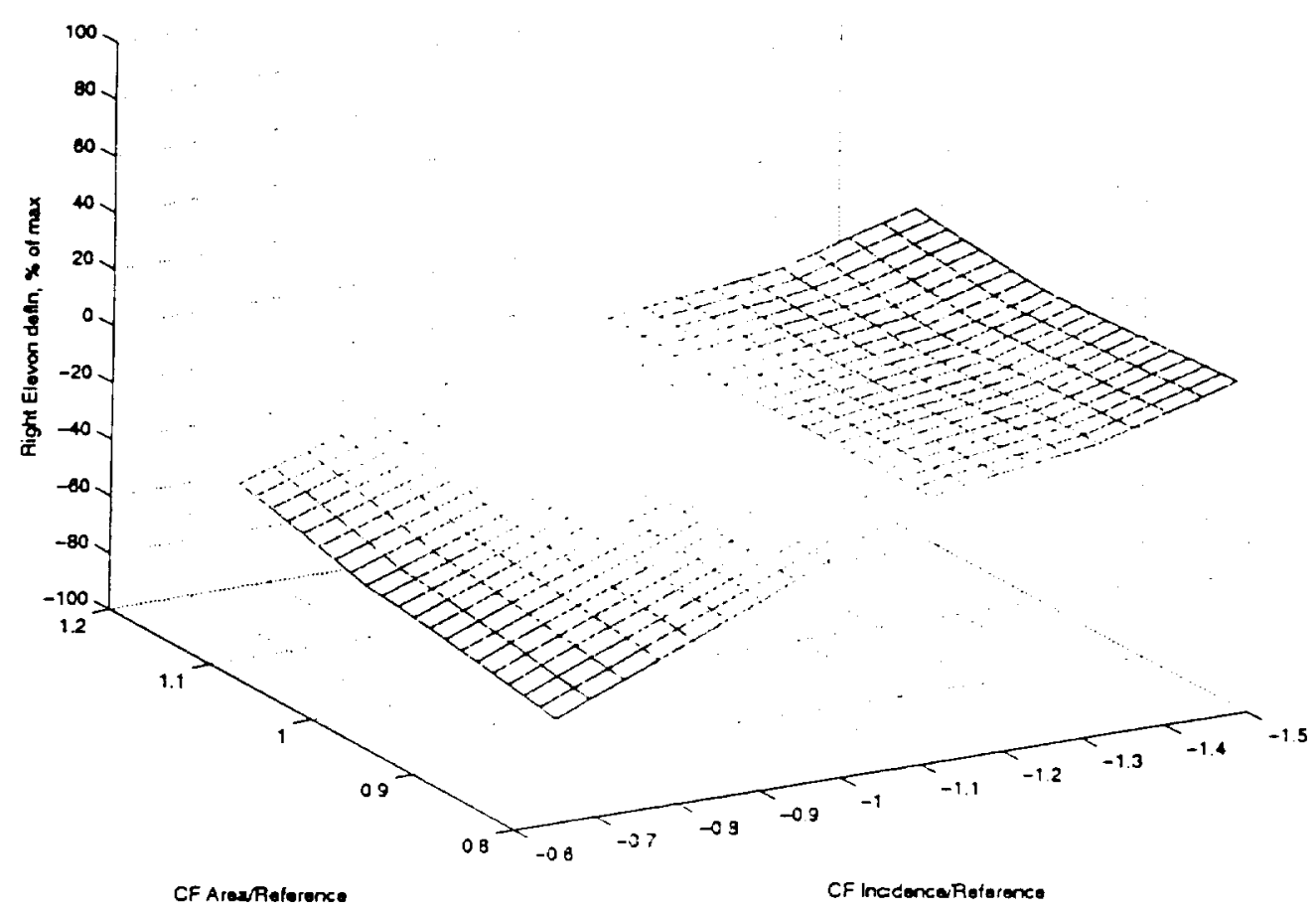

Figure 11 - Right Elevon Deflections as Function of Canted Fin Area and Incidence at $\mathrm{Mach}=1.15$ and Alpha $=3.66 \mathrm{deg}$

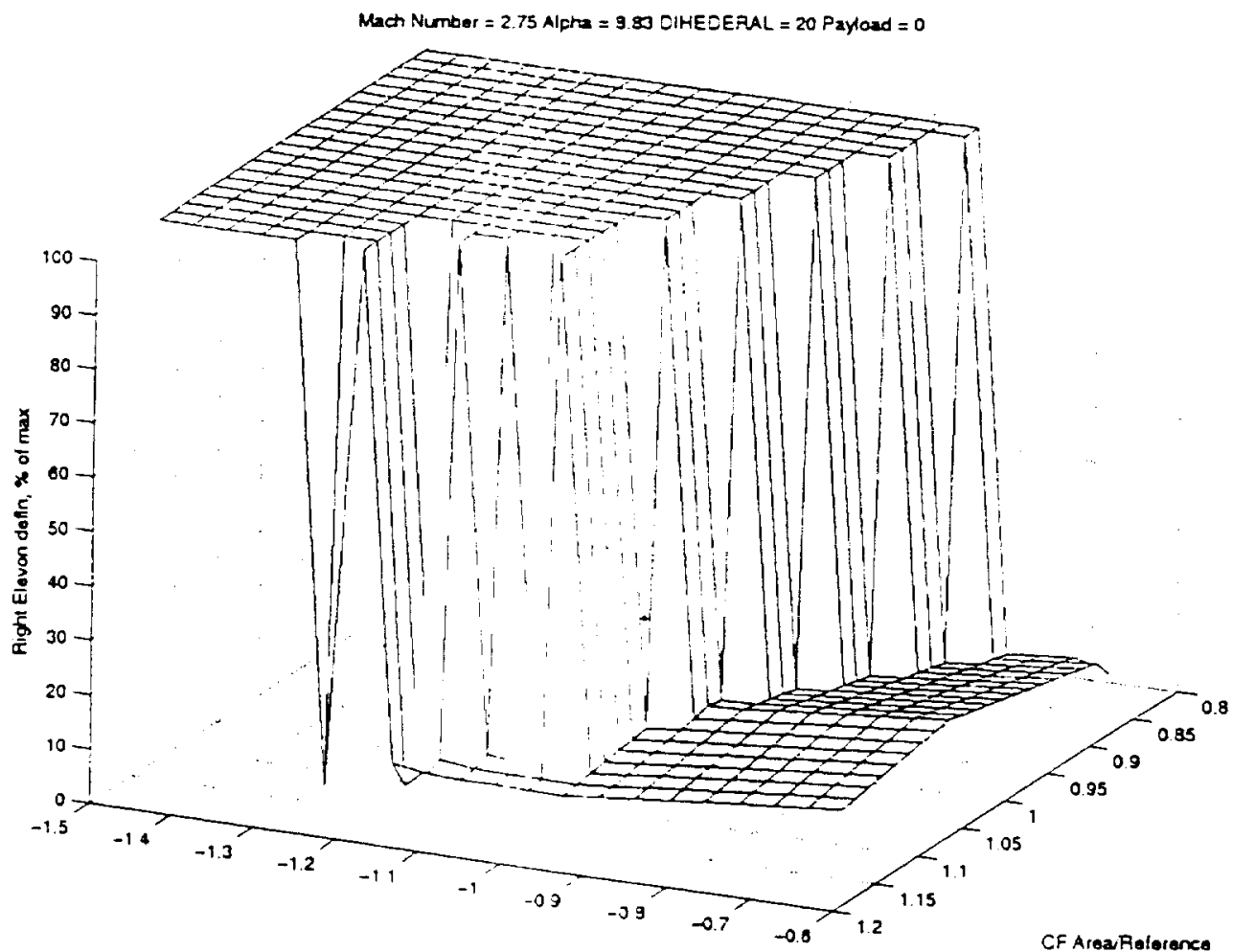

CF incidanceif atoience

Figure 12 - Right Elevon Deflections as Function of Canted Fin Area and Incidence at Mach=2.75 and Alpha $=\mathbf{8 . 8 3} \mathrm{deg}$

American Institute of Aeronautics and Astronautics 


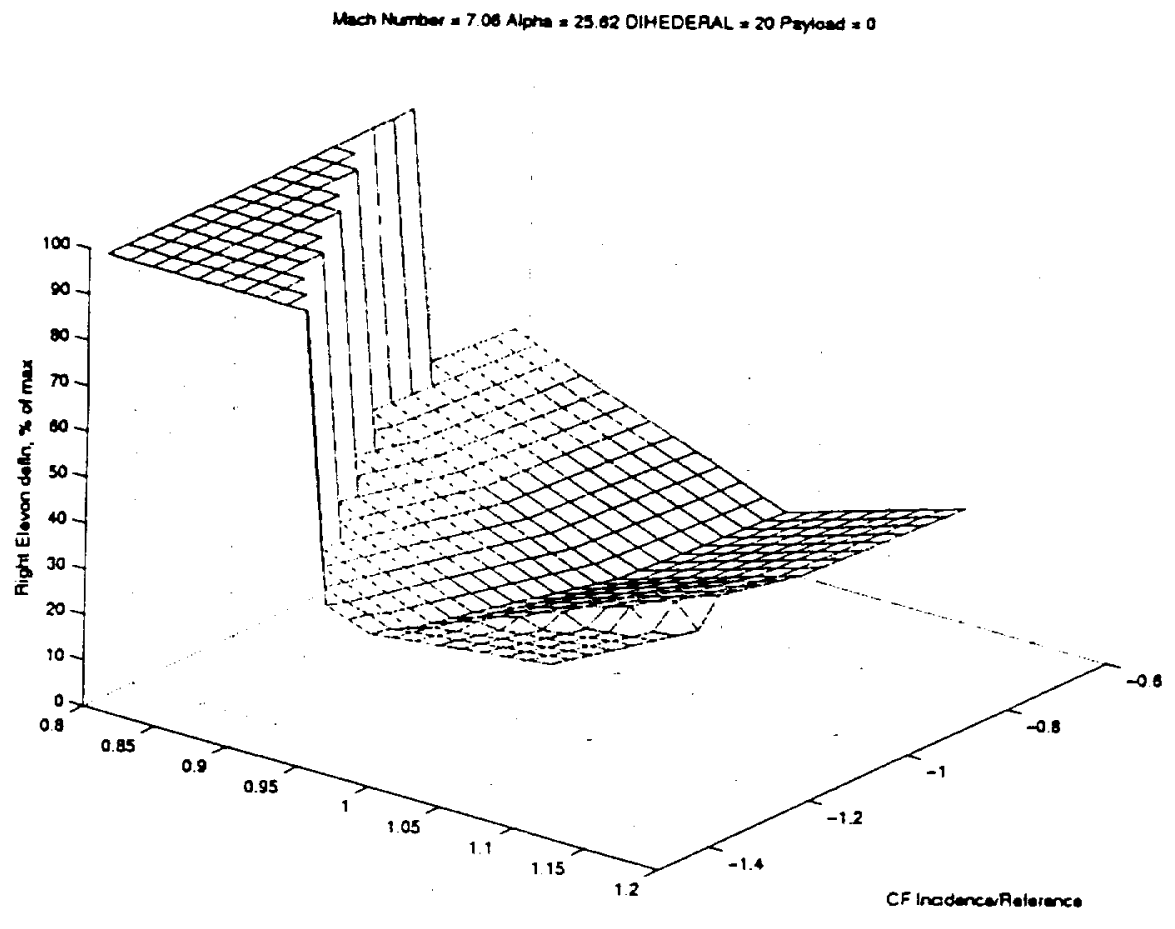

Figure 13 - Right Elevon Deflections as Function of Canted Fin Area and Incidence at Mach=7.06 and Alpha =25.62 deg

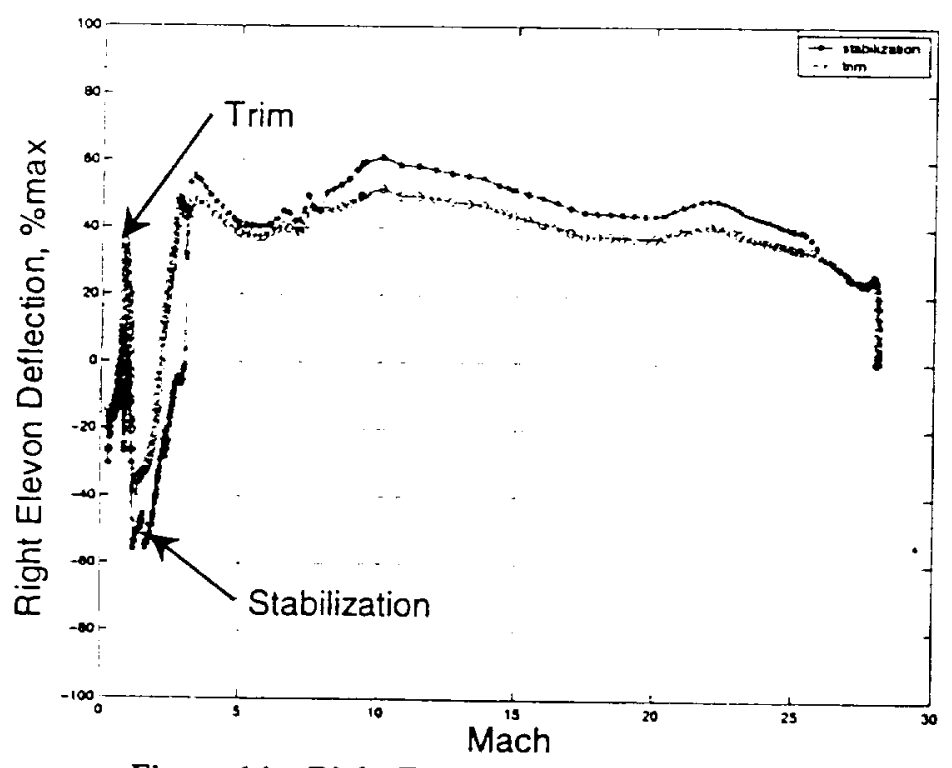

Figure 14 - Right Elevon Deflections Used for Trim and Stabilization for the Final Optimized Configuration

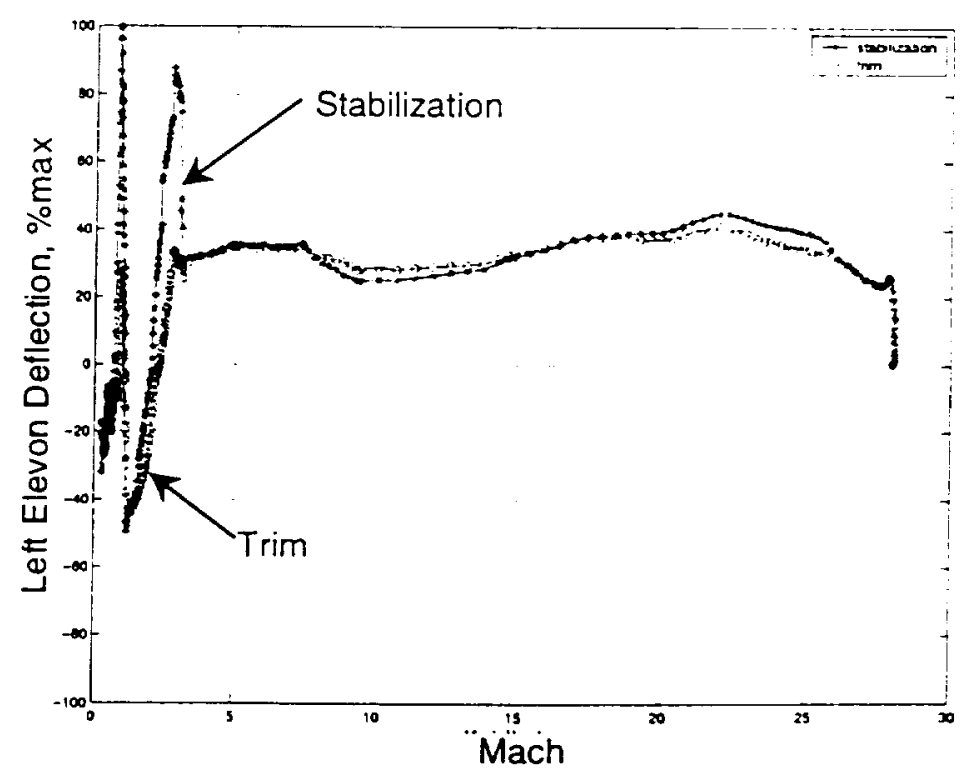

Figure 15 - Left Elevon Deflections Used for Trim and Stabilization for the Final Optimized Configuration 


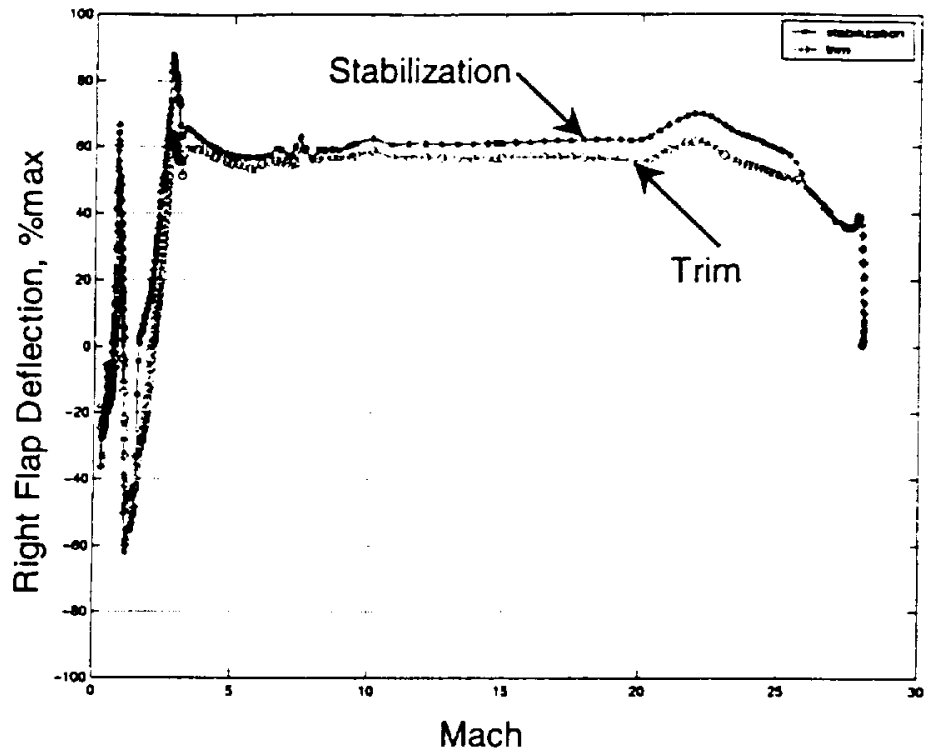

Figure 16 - Right Flap Deflections Used for Trim and Stabilization for the Final Optimized Configuration

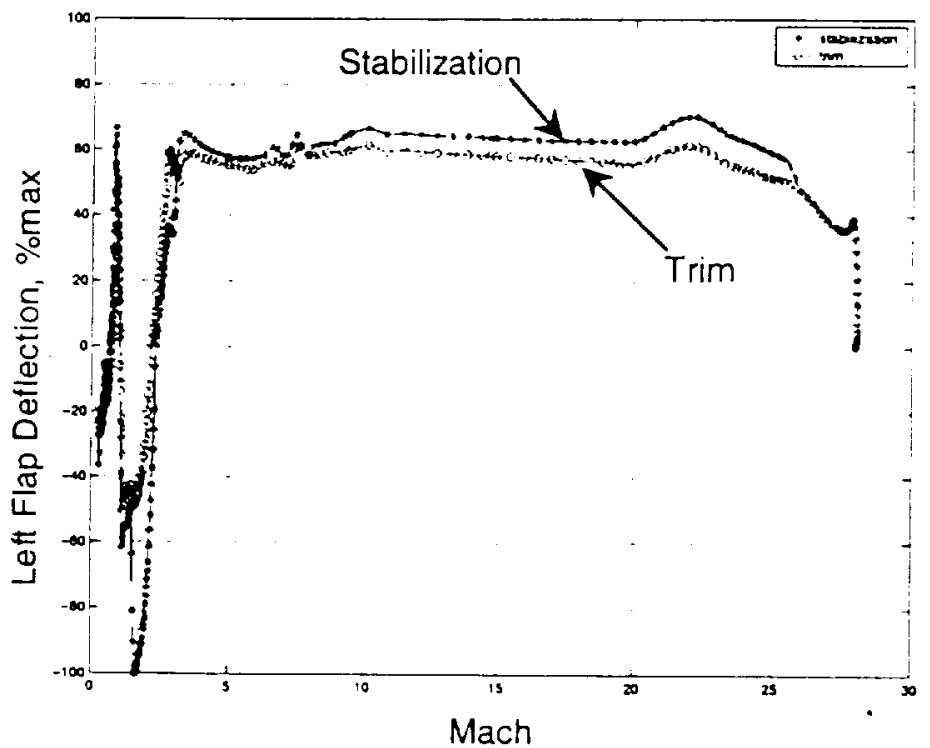

Figure 17 - Left Flap Deflections Used for Trim and Stabilization for the Final Optimized Configuration

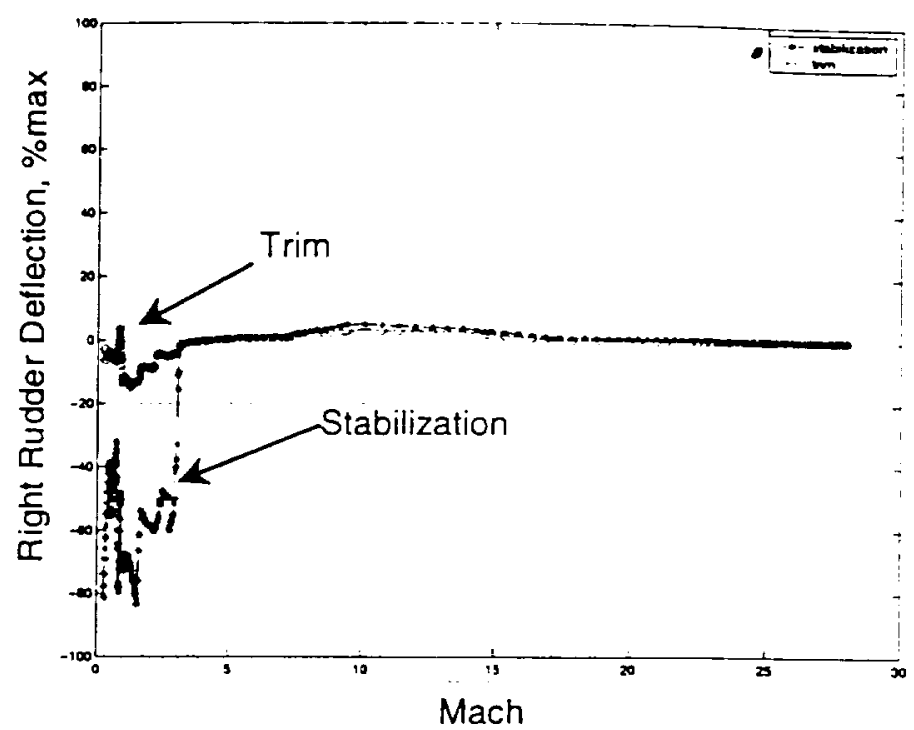

Figure 18 - Right Rudder Deflections Used for Trim and Stabilization for the Final Optimized Configuration

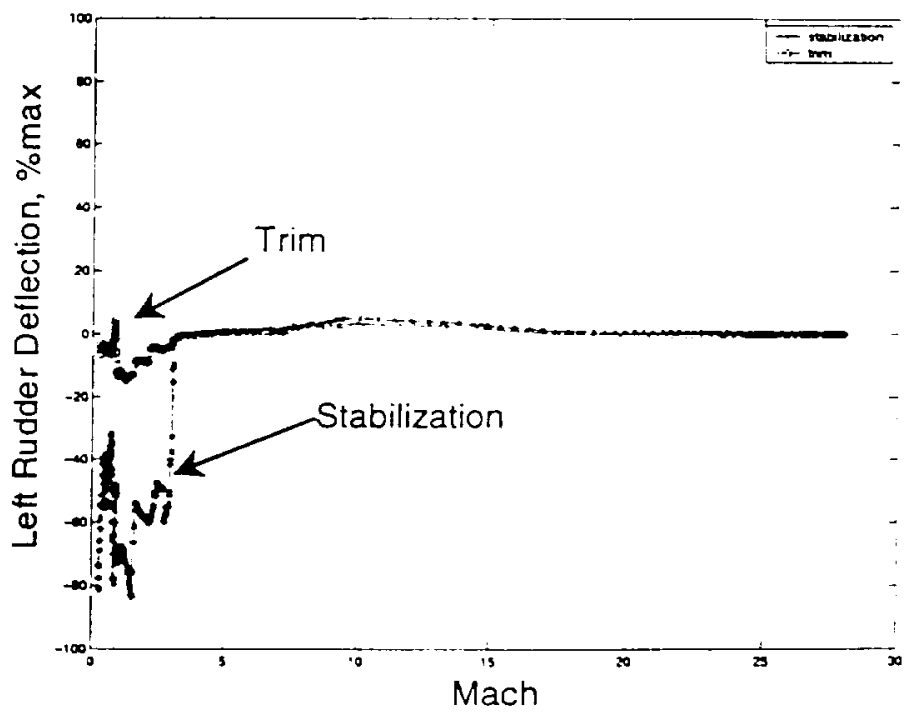

Figure 19 - Left Rudder Deflections Used for Trim and Stabilization for the Final Optimized Configuration 